\title{
Sizable time-reversal violating effects in bottom baryon decays
}

\author{
Chia-Wei Liu and Chao-Qiang Geng \\ School of Fundamental Physics and Mathematical Sciences, \\ Hangzhou Institute for Advanced Study, UCAS, \\ Hangzhou 310024, China \\ E-mail: ron1a2l3d4@gmail.com, cqgeng@ucas.ac.cn
}

\begin{abstract}
We study time-reversal (T) asymmetries in the charmless two-body decays of antitriplet bottom baryons. We find that in $\mathbf{B}_{b} \rightarrow \mathbf{B}_{n} P$ with $\mathbf{B}_{b}=\left(\Xi_{b}^{-}, \Xi_{b}^{0}, \Lambda_{b}\right)$ and $\mathbf{B}_{n}(P)$ a octet baryon (pseudoscalar meson), the positive and negative helicity amplitudes are both sizable, resulting in the large T-violating effects. Particularly, the T-odd parameters, $\beta_{w}$, for the color-enhanced channels are expected to be around $-40 \%$ and $25 \%$ in $b \rightarrow s$ and $b \rightarrow$ $d$ transitions for the standard model, which can be measured by the experiments at LHCb, respectively. On the other hand, in $\mathbf{B}_{b} \rightarrow \mathbf{B}_{n} V(\gamma)$ with $V(\gamma)$ a vector meson (photon), the decays are predominated by a single helicity amplitude, and the $\mathrm{T}$ violating effects by the correlations among spins are suppressed. We also explore the $\mathrm{T}$ violating observables for the $\Xi_{b}$ decays based on the angular distributions.
\end{abstract}

KeYwords: CP violation, Heavy Quark Physics

ArXiv EPrint: 2111.02091 


\section{Contents}

1 Introduction 1

2 T-odd observables 2

$\begin{array}{lll}3 & \text { Cascade decay angular distributions } & 7\end{array}$

$\begin{array}{llr}4 & \text { Conclusions } & 9\end{array}$

\section{Introduction}

It is well known that time-reversal $(\mathrm{T})$ and $\mathrm{CP}$ violations are equivalent due to the $\mathrm{CPT}$ theorem [1]. The indirect and direct evidences of CP violation (CPV) were found in 1964 [2] and $2001[3,4]$, respectively. On the other hand, the first direct evidence on $\mathrm{T}$ violation (TV) was long-awaited until 2012 via the $B^{0}-\bar{B}^{0}$ oscillation [5]. As baryon-antibaryon oscillations are forbidden, a direct measurement on TV in baryon decays is still lacking. Nevertheless, we can study TV with the triple-vector product asymmetries (TPAs), which can be viewed as indirect evidences on TV. The TPAs have been intensively studied in the mesonic weak decays [6-24]. In terms of angular analyses, there are over 40 experimental values related to TPAs in $B \rightarrow V V^{\prime}$ with $V^{(\prime)}=\left(\rho, \omega, K^{*}, \phi\right)$ [25-29]. However, there is still no experimental evidence on a TPA in the bottom baryon decays. Our goal in this work is to seek some measurable large T violating effects (TVEs) in the bottom baryon decays.

Compared to the spinless mesons, the antitriplet bottom baryons $\left(\mathbf{B}_{b}\right)$ are polarized, providing a possibility for observing TPAs even in the two body decays of $\mathbf{B}_{b} \rightarrow \mathbf{B}_{n}(P, V, \gamma)$ with $\mathbf{B}_{n}, P(V)$ and $\gamma$ being the octet baryon, pseudoscalar (vector) meson and photon, respectively. Recently, the lifetimes of $\mathbf{B}_{b}$ have been updated with high precision at LHCb [29-32], and CP violating effects have been studied [33-35]. In particular, a full angular analysis has been carried out in $\Lambda_{b} \rightarrow J / \psi \Lambda$ [36-38]. Clearly, the experimental progresses on $\mathbf{B}_{b}$ result in a great opportunity for us to explore TV in the two-body decays of $\mathbf{B}_{b}$. On the theoretical side for $\mathbf{B}_{b}$ decays, many efforts have been made [39-54], but only few of them have discussed TVEs [55-62]. For the weak transitions described by the effective Hamiltonian $\left(\mathcal{H}_{\text {eff }}\right)$ [63], based on the Hermitian nature of $\mathcal{H}_{\text {eff }}$ and T-symmetry, we have the complex phase approach with

$$
H_{\lambda}=\left\langle\lambda ; \text { "out" }\left|\mathcal{H}_{\text {eff }}\right| \mathbf{B}_{b}\right\rangle=\left\langle\mathbf{B}_{b}\left|\mathcal{H}_{\text {eff }}\right| \lambda ; \text { "in" }\right\rangle,
$$

where $\lambda$ are the helicities of the final states. By ignoring the final state interactions (FSIs), "in" and "out" are interchangable, such that eq. (1.1) demands $H_{\lambda}$ to be real due to the anti-liner property of the T-transformation. This approach is a common method in exploring TV, which has been shown closely related to the TPAs involving momentum and 
spin [55-62]. However, there are some defects in the complex phase approach. The relation between phases and TV is not intuitively understandable. In addition, the analyses of the TPAs carried out in the literature suffer a theoretical inconsistency [62].

In this study, we use a different approach to define an asymmetry parameter with a T-odd scalar operator $\hat{T}$ in the $\mathbf{B}_{b}$ decays, given by

$$
\Delta_{\hat{T}} \equiv \frac{\Gamma\left(\lambda_{t}>0\right)-\Gamma\left(\lambda_{t}<0\right)}{\Gamma\left(\lambda_{t}>0\right)+\Gamma\left(\lambda_{t}<0\right)}
$$

where $\lambda_{t}$ are the eigenvalues of $\hat{T}$. If $\mathcal{H}_{\text {eff respects }}$-symmetry, $\pm \lambda_{t}$ are related by

$$
\mid\left.\left\langle\lambda_{t} ; \text { "out" }\left|\mathcal{H}_{\text {eff }}\right| \mathbf{B}_{b}\right\rangle\right|^{2}-\mid\left.\left\langle\mathbf{B}_{b}\left|\mathcal{H}_{\text {eff }}\right|-\lambda_{t} ; " \text { in" }\right\rangle\right|^{2}=0
$$

Note that if "in" and "out" are interchangable, the left-handed side of eq. (1.3) is proportional to the numerator in $\Delta_{\hat{T}}$, leading to that a nonzero value of $\Delta_{\hat{T}}$ would be a signal of TV. Nonetheless, under FSIs, we have

$$
\left.\left\langle\mp \lambda_{t} ; \text { "out" }\right| \pm \lambda_{t} ; \text { "in" }\right\rangle \neq 0
$$

describing the rescattering effects between $\pm \lambda_{t}$. In this case, $\Delta_{\hat{T}}$ is referred to as a naive Todd observable. To subtract the effects of FSIs, we utilize the CPT symmetry and thereby define a true $\mathrm{T}$ violating observable, which is also a $\mathrm{CP}$ violating one, as [19, 61]

$$
\mathcal{T} \equiv\left(\Delta_{\hat{T}}-( \pm) \bar{\Delta}_{\hat{T}}\right) / 2
$$

where the overline denotes the charge conjugate and the sign corresponds to the parity of $\hat{T}$. The most simple cases for $\hat{T}$ are the triple products of the spins $(\vec{s})$ and 3-momenta $(\vec{p})$ of the particles in the decays. Since there is only one independent 3-momentum in the two-body decays of $\mathbf{B}_{b} \rightarrow \mathbf{B}_{n}(P, V, \gamma), \hat{T}$ contains at least two spins, called as the spin correlations. Consequently, to observe a TVE, a decay must involve at least two different helicities. In this work, we explore TV by concentrating on the T violating observables in $\mathbf{B}_{b} \rightarrow \mathbf{B}_{n} M$.

This paper is organized as follows. In section II, we discuss TV in the two-body decays of $\mathbf{B}_{b} \rightarrow \mathbf{B}_{n}(P, V, \gamma)$. We also provide the numerical results in the standard model for $\mathbf{B}_{b} \rightarrow \mathbf{B}_{n} P$. In section III, we study the angular distributions of the $\mathbf{B}_{b}$ decays. We give our conclusions in section IV.

\section{T-odd observables}

For the $\mathbf{B}_{b}$ decays, the effective Hamiltonian is given by [63]

$$
\mathcal{H}_{\mathrm{eff}}=\frac{G_{F}}{\sqrt{2}} \sum_{f=d, s}\left[V_{u b} V_{u f}^{*}\left(C_{1} O_{1}^{f}+C_{2} O_{2}^{f}\right)-V_{t b} V_{t q}^{*} \sum_{i=3}^{10} C_{i} O_{i}^{f}\right],
$$


with

$$
\begin{aligned}
O_{1}^{f} & =\left(\bar{u}_{\alpha} \gamma^{\mu} L b_{\alpha}\right)\left(\bar{f}_{\beta} \gamma_{\mu} L u_{\beta}\right), & O_{2}^{f} & =\left(\bar{u}_{\alpha} \gamma^{\mu} L b_{\beta}\right)\left(\bar{f}_{\beta} \gamma_{\mu} L u_{\alpha}\right), \\
O_{3}^{f} & =\left(\bar{f}_{\alpha} \gamma^{\mu} L b_{\alpha}\right) \sum_{q}\left(\bar{q}_{\beta} \gamma_{\mu} L q_{\beta}\right), & O_{4}^{f} & =\left(\bar{f}_{\alpha} \gamma^{\mu} L b_{\beta}\right) \sum_{q}\left(\bar{q}_{\beta} \gamma_{\mu} L q_{\alpha}\right), \\
O_{5}^{f} & =\left(\bar{f}_{\alpha} \gamma^{\mu} L b_{\alpha}\right) \sum_{q}\left(\bar{q}_{\beta} \gamma_{\mu} R q_{\beta}\right), & O_{6}^{f} & =\left(\bar{f}_{\alpha} \gamma^{\mu} L b_{\beta}\right) \sum_{q}\left(\bar{q}_{\beta} \gamma_{\mu} R q_{\alpha}\right), \\
O_{7}^{f} & =\frac{3}{2}\left(\bar{f}_{\alpha} \gamma^{\mu} L b_{\alpha}\right) \sum_{q} e_{q}\left(\bar{q}_{\beta} \gamma_{\mu} R q_{\beta}\right), & O_{8}^{f} & =\frac{3}{2}\left(\bar{f}_{\alpha} \gamma^{\mu} L b_{\beta}\right) \sum_{q} e_{q}\left(\bar{q}_{\beta} \gamma_{\mu} R q_{\alpha}\right), \\
O_{9}^{f} & =\frac{3}{2}\left(\bar{f}_{\alpha} \gamma^{\mu} L b_{\alpha}\right) \sum_{q} e_{q}\left(\bar{q}_{\beta} \gamma_{\mu} L q_{\beta}\right), & O_{10}^{f} & =\frac{3}{2}\left(\bar{f}_{\alpha} \gamma^{\mu} L b_{\beta}\right) \sum_{q} e_{q}\left(\bar{q}_{\beta} \gamma_{\mu} L q_{\alpha}\right), \\
O_{7 \gamma}^{f} & =\frac{e}{8 \pi^{2}} m_{\mathrm{b}} \bar{f}_{\alpha} \sigma^{\mu \nu} R b_{\alpha} F_{\mu \nu}, & O_{8 G} & =\frac{g}{8 \pi^{2}} m_{\mathrm{b}} \bar{f}_{\alpha} \sigma^{\mu \nu} T_{\alpha \beta}^{a} R b_{\beta} G_{\mu \nu}^{a},
\end{aligned}
$$

where $O_{1,2}, O_{3,4,5,6}, O_{7,8,9,10}$ and $O_{7 \gamma, 8 G}$ correspond to tree, penguin, electroweak-penguin and magnetic-penguin operators, respectively, $G_{F}$ is the Fermi constant, $V$ represents the CKM quark mixing matrix, $\alpha$ and $\beta$ denote the color, $q=(u, d, s)$, and $L(R)$ stands for the chiral structure of $1 \mp \gamma_{5}$.

In the radiative decays of $\mathbf{B}_{b} \rightarrow \mathbf{B}_{n} \gamma$, the relevant operator is $O_{7 \gamma}^{f}$, in which $f$ is essentially left handed. As the decays are predominated by a single helicity state of $\lambda_{n}=$ $-1 / 2$, the T-violating effects are expected to be suppressed. In the factorization approach for $\mathbf{B}_{b} \rightarrow \mathbf{B}_{n}(P, V), O_{1,2,3,4,9,10}$ can only produce left-handed $\mathbf{B}_{n}$ since they involve only left-handed currents. On the other hand, for $O_{5,6,7,8}$, based on the Fierz transformation, one has that

$$
\left(\bar{f} \gamma^{\mu} L b\right) \sum_{q}\left(\bar{q} \gamma_{\mu} R q\right)=-2(\bar{q} L b)(\bar{f} R q)
$$

in which the chiral structure of $(\bar{q} L b)$ leads to a right-handed $\mathbf{B}_{n}$, given by

$$
\left\langle\mathbf{B}_{n}|\bar{q} L b| \mathbf{B}_{b}\right\rangle=\bar{u}_{n}\left(f_{s}-g_{a} \gamma_{5}\right) u_{b},
$$

where $u_{b(n)}$ represents the Dirac spinor for $\mathbf{B}_{b(n)}$, and $f_{s}\left(g_{a}\right)$ corresponds to the (pseudo)scalar form factor with $f_{s}=g_{a}$ in the heavy quark limit. Here, the color index has not been explicitly shown. Clearly, the amplitudes between $\lambda_{n}=-1 / 2$ and $\lambda_{n}=1 / 2$ from $O_{1,2,3,4,9,10}$ and $O_{5,6,7,8}$, respectively, can potentially have interferences. Furthermore, in $\mathbf{B}_{b} \rightarrow \mathbf{B}_{n} V$, the vector meson matrix elements of (pseudo)scalar operators vanish, read as

$$
\langle V|\bar{f} R q| 0\rangle=0,
$$

resulting in that the TVEs are suppressed because the decays are dominated by a single helicity state [62]. In contrast, in $\mathbf{B}_{b} \rightarrow \mathbf{B}_{n} P$, the pseudoscalar meson matrix elements are enhanced by the smallness of the light quark masses, given by

$$
i\langle P|\bar{f} R q| 0\rangle=\frac{m_{P}^{2}}{m_{f}+m_{q}} f_{P},
$$

with $m$ standing for the mass and $f_{P}$ the meson decay constants. Since the denominators of the right side of eq. (2.6) are made of the light quark masses, $O_{5,6}$ cause a significant 
contribution to $\lambda_{n}=1 / 2$, providing a interference with $\lambda_{n}=-1 / 2$ from $O_{1,2}$. We conclude that the decays of $\mathbf{B}_{b} \rightarrow \mathbf{B}_{n} P$ are the most promising modes to observe TVEs.

In $\mathbf{B}_{b} \rightarrow \mathbf{B}_{n} P$, as $P$ are spinless, the spin correlations only happen between $\mathbf{B}_{b}$ and $\mathbf{B}_{n}$. The most simple T-odd observables are then described by

$$
\hat{T} \equiv(\hat{p} \times \vec{s}) \cdot \vec{J}
$$

where $\hat{p}(\vec{s})$ are the 3 -momenta (spins) of $\mathbf{B}_{n}$, and $\vec{J}$ are the angular momenta in the final states. Since $\vec{J}$ are always conserved, one can identify them as the spins of $\mathbf{B}_{b}$. The eigenstates of $\hat{T}$ are related to the helicity ones as [62]

$$
\left|\lambda_{t}= \pm \frac{1}{2}\right\rangle=\frac{1}{\sqrt{2}}\left(\left|H_{+}\right\rangle \pm i\left|H_{-}\right\rangle\right)
$$

with $J=1 / 2$ in both sides. The naive T-odd observable is then given by the asymmetry between $\lambda_{t}= \pm 1 / 2$, read as [64]

$$
\beta \equiv \frac{\Gamma\left(\lambda_{t}=\frac{1}{2}\right)-\Gamma\left(\lambda_{t}=-\frac{1}{2}\right)}{\Gamma\left(\lambda_{t}=\frac{1}{2}\right)+\Gamma\left(\lambda_{t}=-\frac{1}{2}\right)}=\frac{2 \Im\left(H_{+} H_{-}^{*}\right)}{\left|H_{+}\right|^{2}+\left|H_{-}\right|^{2}}=\frac{2 \Im\left(\mathcal{P} \mathcal{S}^{*}\right)}{|\mathcal{S}|^{2}+|\mathcal{P}|^{2}},
$$

where $\mathcal{S}$ and $\mathcal{P}$ are the amplitudes with negative and positive parities, respectively, and $H_{ \pm}$stand for the amplitudes with $\lambda_{n}= \pm 1 / 2$. Thereby, from eq. (1.5), we can define the true and fake T-odd observables as [61]

$$
\beta_{w}=(\beta+\bar{\beta}) / 2, \quad \beta_{s}=(\beta-\bar{\beta}) / 2,
$$

related by the CP-odd and even phases, respectively. We emphasize that a nonzero value of $\beta_{w}$ implies not only TV but also CPV. To have a complete study in $\mathbf{B}_{b} \rightarrow \mathbf{B}_{n} P$, we also define the direct $\mathrm{CP}$ and up-down asymmetries by [64]

$$
\begin{aligned}
A_{\mathrm{CP}} & \equiv \frac{\Gamma-\bar{\Gamma}}{\Gamma+\bar{\Gamma}}, \\
\alpha_{b} & \equiv \frac{\Gamma\left(\lambda_{n}=\frac{1}{2}\right)-\Gamma\left(\lambda_{n}=-\frac{1}{2}\right)}{\Gamma\left(\lambda_{n}=\frac{1}{2}\right)+\Gamma\left(\lambda_{n}=-\frac{1}{2}\right)}=\frac{2 \Re\left(\mathcal{P} \mathcal{S}^{*}\right)}{|\mathcal{S}|^{2}+|\mathcal{P}|^{2}},
\end{aligned}
$$

respectively. The linear combinations of $\alpha_{b}$ and $\bar{\alpha}_{b}$ with the overline denoting the charge conjugate are given by

$$
\alpha_{\mathrm{av}} \equiv\left(\alpha_{b}-\bar{\alpha}_{b}\right) / 2, \quad \Delta \alpha=\frac{\alpha_{b}+\bar{\alpha}_{b}}{\alpha_{b}-\bar{\alpha}_{b}},
$$

in which $\Delta \alpha$ is a CP-odd quantity.

It is well known that to have nonzero values of $A_{\mathrm{CP}}$ and $\Delta \alpha$, nonvanishing strong phases are both required. Explicitly, one has that

$$
A_{\mathrm{CP}}, \Delta \alpha \sim \sin \phi_{s} \sin \phi_{w},
$$



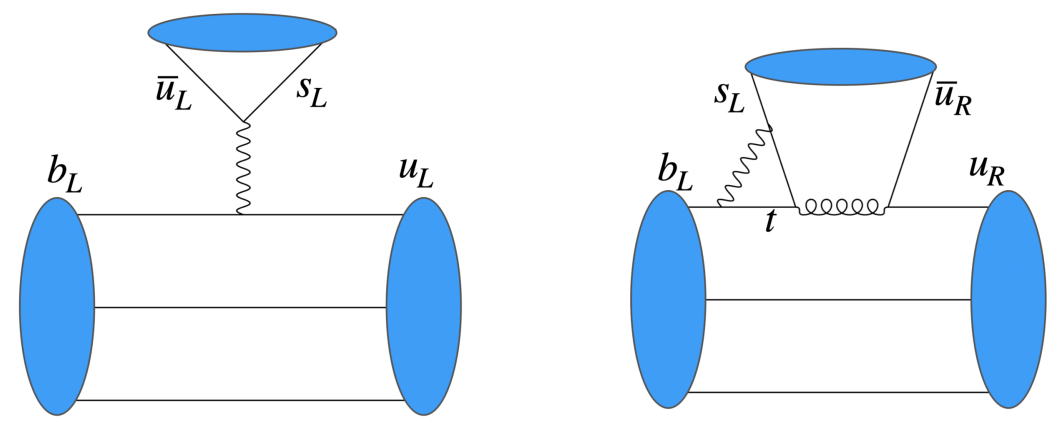

Figure 1. Topological diagrams for the color enhanced processes in $\mathbf{B}_{b} \rightarrow \mathbf{B}_{n} P$, where the left and right figures are associated with the CKM coefficients of $V_{u b} V_{u s}^{*}$ and $V_{t b} V_{t s}^{*}$, respectively.

where $\phi_{s}$ and $\phi_{w}$ correspond to the CP-even and CP-odd phases between $\mathcal{S}$ and $\mathcal{P}$, respectively. On the other hand, one can show that

$$
\beta_{w} \propto \cos \phi_{s} \sin \phi_{w}, \quad \beta_{s} \propto \cos \phi_{w} \sin \phi_{s} .
$$

Clearly, a nonzero strong phase is not necessary in $\beta_{w}$. In fact, when $\phi_{s} \rightarrow 0, \beta_{w}$ becomes maximal, whereas $\left(A_{\mathrm{CP}}, \Delta \alpha\right) \rightarrow 0$. Since the strong phases are much less understood compared to the weak ones, $\beta_{w}$ could be a more promising $\mathrm{T}$ and $\mathrm{CP}$ violating observable compared to the others.

In $\mathbf{B}_{b} \rightarrow \mathbf{B}_{n} P$, TV depends on the relative CP-odd phase between $V_{u b} V_{u f}^{*}$ and $V_{t b} V_{t f}^{*}$ in the different helicity amplitudes (see eq. (2.9) and figure 1), related to the interferences between $O_{1,2}^{f}$ and $O_{5,6}^{f}$. Since $C_{1,6}$ are much larger compared to $C_{2,5}$, respectively, one expects that large $\mathrm{T}$-asymmetries can be induced in the color-enhanced decays.

In practice, the decays are often dominated by one of the helicities. For $\left|H_{ \pm}\right|^{2} /\left|H_{\mp}\right|^{2} \gg$ 0 , we have the approximations, given by

$$
\beta \approx 2 \Im\left(\frac{H_{+} H_{-}^{*}}{H_{ \pm} H_{ \pm}^{*}}\right)=\mp 2 \Im\left(\frac{H_{\mp}}{H_{ \pm}}\right)
$$

As we will see subsequently, in $\mathbf{B}_{b} \rightarrow \mathbf{B}_{n} P$, the transitions associated with $b \rightarrow s$ and $b \rightarrow d$ are predominated by $H_{+}$and $H_{-}$, respectively. Using the estimations of $H_{-} \propto c_{1} V_{u b} V_{u f}^{*}$ and $H_{+} \propto-c_{6} V_{t b} V_{t f}^{*}$, we have

$$
\beta_{w} \approx 2 \Im\left(\frac{V_{u b} V_{u s}^{*} c_{1}}{V_{t b} V_{t s}^{*} c_{6}}\right)=\frac{2 c_{1}}{c_{6}} \lambda^{2} \eta \approx-0.7
$$

and

$$
\beta_{w} \approx-2 \Im\left(\frac{V_{t b} V_{t d}^{*} c_{6}}{V_{u b} V_{u d}^{*} c_{1}}\right) \approx-\frac{2 c_{6}}{c_{1}} \frac{\eta}{\rho^{2}+\eta^{2}} \approx 0.2
$$

for $b \rightarrow s$ and $b \rightarrow d$ transitions, respectively. Here, we have used $c_{6} / c_{1}=0.04$ [63] and the Wolfenstein parametrization for the CKM matrix, given by [65]

$$
\left[\begin{array}{lll}
V_{u d} & V_{u s} & V_{u b} \\
V_{c d} & V_{c s} & V_{c b} \\
V_{t d} & V_{t s} & V_{t b}
\end{array}\right]=\left[\begin{array}{ccc}
1-\lambda^{2} / 2 & \lambda & A \lambda^{3}(\rho-i \eta) \\
-\lambda & 1-\lambda^{2} / 2 & A \lambda^{2} \\
A \lambda^{3}(1-\rho-i \eta) & -A \lambda^{2} & 1
\end{array}\right]+O\left(\lambda^{4}\right)
$$


It is interesting to point out that although $\left|V_{u b} V_{u s}^{*} / V_{t b} V_{t s}^{*}\right|$ and $\left|V_{u b} V_{u d}^{*} / V_{t b} V_{t d}^{*}\right|$ are very different, $\left|\beta_{w}\right|$ are in the same size for $b \rightarrow s$ and $b \rightarrow d$ transitions. It is due to that they are applied at the different limits of eq. (2.16), allowing them to be both sizable.

It is known that the factorization approach works well in the color-enhanced channels [66]. To get the more precise results, we adopt the generalized factorization approach, in which the renormalization dependency in the naive factorization has been absorbed into the effective Wilson coefficients to the next to leading log precision [66]. Accordingly, the color-enhanced amplitudes are given as [42, 43, 48, 54]

$$
\left\langle\mathbf{B}_{n} P\left|\mathcal{H}_{\mathrm{eff}}\right| \mathbf{B}_{b}\right\rangle=i \bar{u}_{n}\left(A+B \gamma_{5}\right) u_{b},
$$

with

$$
\begin{aligned}
& A=\frac{G_{F}}{\sqrt{2}} f_{P}\left\{m_{b} V_{u b} V_{u f}^{*} a_{1}-V_{t b} V_{t f}^{*}\left[m_{b}\left(a_{4}+a_{10}\right)+\frac{2 m_{P}^{2}}{m_{u}+m_{f}}\left(a_{6}+a_{8}\right)\right]\right\} f_{s}, \\
& B=\frac{G_{F}}{\sqrt{2}} f_{P}\left\{m_{b} V_{u b} V_{u f}^{*} a_{1}-V_{t b} V_{t f}^{*}\left[m_{b}\left(a_{4}+a_{10}\right)-\frac{2 m_{P}^{2}}{m_{u}+m_{f}}\left(a_{6}+a_{8}\right)\right]\right\} g_{a},
\end{aligned}
$$

where $a_{i}$ are the effective Wilson coefficients [42, 43, 54]. The helicity amplitudes with definite angular momenta are then given as [67]

$$
H_{ \pm}=\sqrt{Q_{+}} A \mp \sqrt{Q_{-}} B
$$

with $Q_{ \pm}=\sqrt{\left(m_{\mathbf{B}_{b}} \pm m_{\mathbf{B}_{n}}\right)^{2}-m_{P}^{2}}$, and the decay widths are given by

$$
\Gamma=\frac{\left|\vec{p}_{c}\right|}{16 \pi m_{\mathbf{B}_{b}^{2}}}\left(\left|H_{+}\right|^{2}+\left|H_{-}\right|^{2}\right)
$$

where $\vec{p}_{c}$ are the 3-momenta of $\mathbf{B}_{n}$ in the central mass frames. At the heavy quark limit, we have that $Q_{+}=Q_{-}$. It is straight forward to see that the terms associated with $\left(a_{6}+a_{8}\right)$ contribute to $H_{+}$, while the others $H_{-}$only.

We evaluate the form factors of $f_{s}$ and $g_{a}$ in eq. (2.21) from the modified bag model [48] with the numerical results given in table $1,{ }^{1}$ where the first and second uncertainties in the branching ratios come from the bag radius of $R=(4.8 \pm 0.2 \mathrm{GeV})^{-1}[48]$ and CKM elements [29], respectively. In particular, the $\mathrm{T}$ violating asymmetries are found to be

$$
\begin{aligned}
& \beta_{w}\left(\Xi_{b}^{0} \rightarrow \Sigma^{+} K^{-}, \Xi_{b}^{-} \rightarrow \Lambda^{0} K^{-}, \Xi_{b}^{-} \rightarrow \Sigma^{0} K^{-}, \Lambda_{b}^{0} \rightarrow p K^{-}\right) \\
& \quad=-0.38 \pm 0.01,-0.39 \pm 0.01,-0.38 \pm 0.01,-0.35 \pm 0.01 \\
& \beta_{w}\left(\Xi_{b}^{0} \rightarrow \Sigma^{+} \pi^{-}, \Xi_{b}^{-} \rightarrow \Lambda^{0} \pi^{-}, \Xi_{b}^{-} \rightarrow \Sigma^{0} \pi^{-}, \Lambda_{b}^{0} \rightarrow p \pi^{-}\right) \\
& =0.25 \pm 0.02,0.25 \pm 0.02,0.25 \pm 0.01,0.25 \pm 0.02
\end{aligned}
$$

for $b \rightarrow d$ and $b \rightarrow s$ transitions in the standard model, respectively. As the absolute values of the asymmetries can be as large as $40 \%$ and $25 \%$, they can be measured in the

\footnotetext{
${ }^{1}$ The decay branching ratios in this work are slightly different with those in ref. [48] as we have updated the experimental inputs of the CKM matrix elements.
} 


\begin{tabular}{|c|c|c|c|c|c|}
\hline \multicolumn{2}{|c|}{ channels } & $\begin{array}{l}10^{6} \mathcal{B} \\
A_{C P}\end{array}$ & $\begin{array}{l}\alpha_{\mathrm{av}} \\
\Delta \alpha\end{array}$ & $\beta_{s}$ & $\phi_{s}$ \\
\hline \multirow{4}{*}{$\Xi_{b}^{0} \rightarrow$} & \multirow{2}{*}{$\Sigma^{+} K^{-}$} & $7.52 \pm 1.29 \pm 0.27$ & $0.40 \pm 0.01$ & $-0.04 \pm 0.02$ & $0.15 \pm 0.00$ \\
\hline & & $0.06 \pm 0.00$ & $0.18 \pm 0.00$ & $-0.38 \pm 0.01$ & $0.76 \pm 0.01$ \\
\hline & \multirow{2}{*}{$\Sigma^{+} \pi^{-}$} & $6.62 \pm 1.09 \pm 0.86$ & $-0.93 \pm 0.01$ & $0.08 \pm 0.04$ & $0.08 \pm 0.00$ \\
\hline & & $-0.05 \pm 0.00$ & $-0.01 \pm 0.00$ & $0.25 \pm 0.02$ & $-2.88 \pm 0.02$ \\
\hline \multirow{8}{*}{$\Xi_{b}^{-} \rightarrow$} & \multirow{2}{*}{$\Lambda^{0} K^{-}$} & $1.10 \pm 0.27 \pm 0.04$ & $0.41 \pm 0.01$ & $-0.04 \pm 0.02$ & $0.15 \pm 0.00$ \\
\hline & & $0.06 \pm 0.00$ & $0.18 \pm 0.00$ & $-0.39 \pm 0.01$ & $0.76 \pm 0.01$ \\
\hline & \multirow{2}{*}{$\Sigma^{0} K^{-}$} & $4.00 \pm 0.68 \pm 0.14$ & $0.40 \pm 0.01$ & $-0.04 \pm 0.02$ & $0.15 \pm 0.00$ \\
\hline & & $0.06 \pm 0.00$ & $0.18 \pm 0.00$ & $-0.38 \pm 0.01$ & $0.76 \pm 0.01$ \\
\hline & \multirow{2}{*}{$\Lambda^{0} \pi^{-}$} & $0.98 \pm 0.24 \pm 0.13$ & $-0.93 \pm 0.01$ & $0.08 \pm 0.04$ & $0.08 \pm 0.00$ \\
\hline & & $-0.05 \pm 0.00$ & $-0.01 \pm 0.00$ & $0.25 \pm 0.02$ & $-2.88 \pm 0.02$ \\
\hline & \multirow{2}{*}{$\Sigma^{0} \pi^{-}$} & $3.52 \pm 0.58 \pm 0.46$ & $-0.93 \pm 0.01$ & $0.08 \pm 0.00$ & $0.08 \pm 0.00$ \\
\hline & & $-0.05 \pm 0.00$ & $-0.01 \pm 0.00$ & $0.25 \pm 0.02$ & $-2.88 \pm 0.02$ \\
\hline \multirow{4}{*}{$\Lambda_{b}^{0} \rightarrow$} & \multirow{2}{*}{$p K^{-}$} & $5.52 \pm 0.65 \pm 0.19$ & $0.37 \pm 0.01$ & $-0.04 \pm 0.02$ & $0.15 \pm 0.00$ \\
\hline & & $0.06 \pm 0.00$ & $0.18 \pm 0.00$ & $-0.35 \pm 0.01$ & $0.76 \pm 0.01$ \\
\hline & \multirow{2}{*}{$p \pi^{-}$} & $4.62 \pm 0.52 \pm 0.60$ & $-0.91 \pm 0.006$ & $0.09 \pm 0.04$ & $0.08 \pm 0.00$ \\
\hline & & $-0.05 \pm 0.00$ & $-0.03 \pm 0.00$ & $0.25 \pm 0.02$ & $-2.88 \pm 0.02$ \\
\hline
\end{tabular}

Table 1. Numerical results in $\mathbf{B}_{b} \rightarrow \mathbf{B}_{n} P$, where the first and second uncertainties in the branching ratios come from the bag radius [48] and CKM elements [29], respectively.

experiments at $\mathrm{LHCb}$, which provide a great opportunity to directly observe TV. The main differences compared to the naive estimations in eqs. (2.17) and (2.18) are caused by the corrections from the effective Wilson coefficients, given by $\left|a_{6} / c_{6}\right| \approx 1.5[42,43,48,54,66]$. Note that the results of $\alpha$ and $\beta$ have small uncertainties due to the correlations between $f_{s}$ and $g_{a}$, i.e. their ratios depend little on the model parameters. Nonetheless, it must be emphasized that the uncertainties from the nonfactorizable effects are not considered as they can not be reliably calculated. However, the $\Xi_{b}^{-}$decays are free from the W-exchange diagrams, making the results reliable.

\section{Cascade decay angular distributions}

We now concentrate on the effects of the T-odd spin correlations. Although the cascade decays of $\mathbf{B}_{n}$ conserve T-symmetry, the T-odd spin correlations in $\mathbf{B}_{b} \rightarrow \mathbf{B}_{n} P$ are handed down to the angular distributions of the sequential particles. For the level-2 cascade decays of $\Xi_{b}^{-} \rightarrow \Lambda\left(\rightarrow p \pi^{-}\right) K^{-} / \pi^{-}$and $\Xi_{b}^{0} \rightarrow \Sigma^{+}\left(\rightarrow p \pi^{0}\right) K^{-} / \pi^{-}$, the angular distributions are given by

$$
\begin{aligned}
\mathcal{D}_{2}(\vec{\Omega}) & =\frac{1}{\Gamma} \frac{\partial^{3} \Gamma}{\partial \cos \theta \partial \cos \theta_{1} \partial \phi} \\
& =\frac{1}{8 \pi}\left[1+P_{b} \alpha \cos \theta \cos \theta_{1}+\alpha_{b}\left(\alpha \cos \theta_{1}+P_{b} \cos \theta\right)+P_{b} \alpha\left(\beta \sin \phi-\gamma^{\prime} \cos \phi\right) \sin \theta \sin \theta_{1}\right],
\end{aligned}
$$




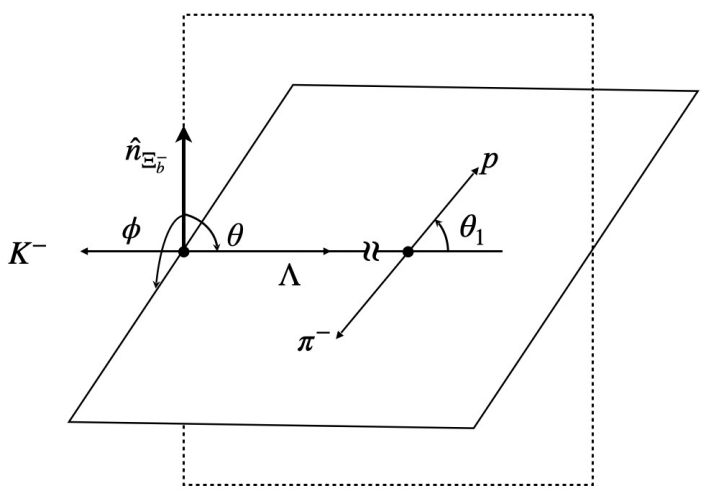

(a) Cascade decays with $\Xi_{b}^{-} \rightarrow \Lambda\left(\rightarrow p \pi^{-}\right) K^{-}$.

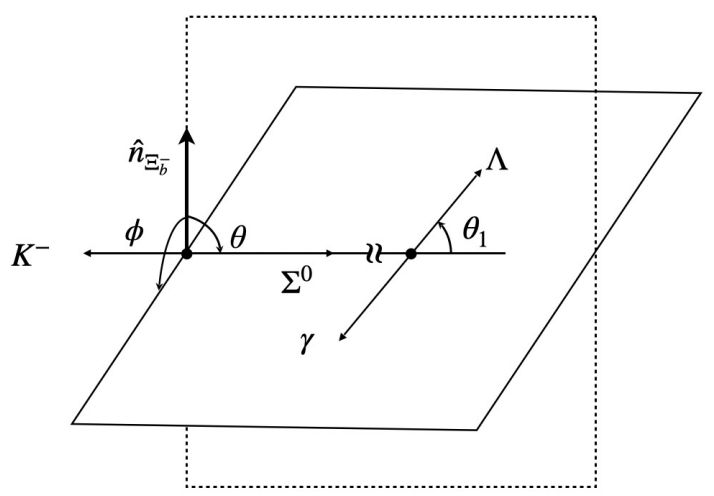

(c) Cascade decays with $\Xi_{b}^{-} \rightarrow \Sigma^{0}(\rightarrow \Lambda \gamma) K^{-}$.

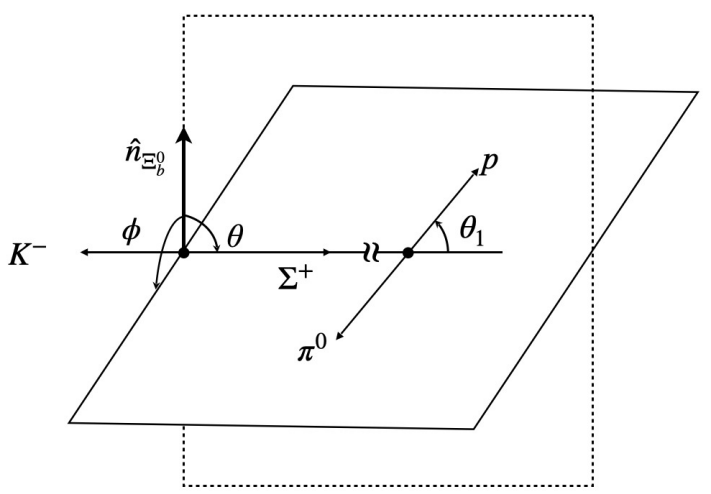

(b) Cascade decays with $\Xi_{b}^{0} \rightarrow \Sigma^{+}\left(\rightarrow p \pi^{0}\right) K^{-}$.

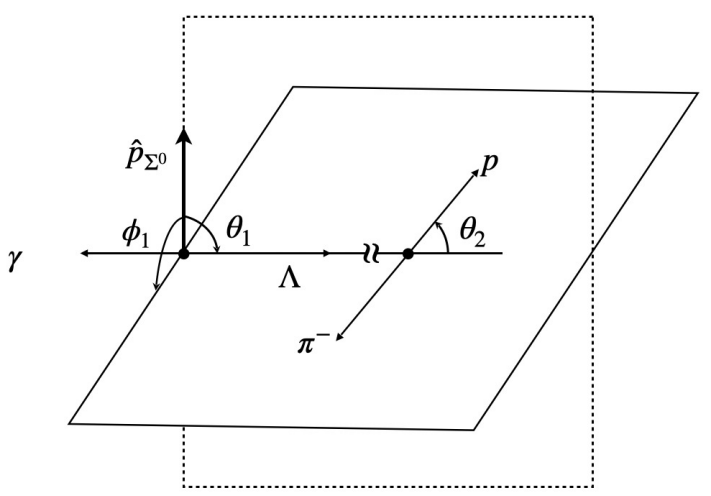

(d) Cascade decays with $\Sigma^{0} \rightarrow \Lambda\left(\rightarrow p \pi^{-}\right) \gamma$.

Figure 2. Angles in $\mathcal{D}(\vec{\Omega})$ for cascade decays.

where $P_{b}$ describe the $\Xi_{b}$ polarization fractions, $\alpha\left(=\alpha_{\Lambda, \Sigma^{+}}\right)$represent the up-down asymmetry parameters in $\Lambda \rightarrow p \pi^{-}$and $\Sigma^{+} \rightarrow p \pi^{0}$, and

$$
\gamma^{\prime} \equiv \frac{|\mathcal{S}|^{2}-|\mathcal{P}|^{2}}{|\mathcal{S}|^{2}+|\mathcal{P}|^{2}}
$$

Here, $\theta$ and $\phi$ stand for the polar and azimuthal angles in the level- 1 decays, while $\theta_{1}$ is the polar angle in the level-2 decays (see figures $2 \mathrm{a}$ and $2 \mathrm{~b}$, in which we have taken $P=K^{-}$ as an example).

We see that the T-odd spin correlations indeed manifest themselves in $\mathcal{D}_{2}(\vec{\Omega})$ and therefore can be observed in the experiments at LHCb. They are described by the asymmetries of the dihedral angles between the initial and cascade decay planes. To probe them, it is sufficient to consider the $\phi$-dependence only. Based on the numbers of events, we define the naive T-odd observable as

$$
\mathcal{T}\left(N_{+}, N_{-}\right)=\frac{N_{+}-N_{-}}{N_{+}+N_{-}}
$$

where $N_{ \pm}$are the numbers of events with $\pi>\phi \geq 0$ and $2 \pi>\phi \geq \pi$, respectively. For sufficient data points, we have

$$
\lim _{N_{ \pm} \rightarrow \infty} \mathcal{T}\left(N+, N_{-}\right)=\frac{1}{\Gamma}\left(\int_{0}^{\pi} \frac{\partial \Gamma}{\partial \phi}-\int_{\pi}^{2 \pi} \frac{\partial \Gamma}{\partial \phi}\right)=\frac{\pi}{8} P_{b} \alpha \beta
$$


Nonetheless, $\beta$ are always followed by $P_{b}$, requiring that $\Xi_{b}$ should be polarized. This is a reasonable result since $\beta$ shall be able to trace back to the spin correlations, and naturally demands that $P_{b} \neq 0$.

On the other hand, $\Xi_{b}^{-} \rightarrow \Sigma^{0}\left(\rightarrow \Lambda\left(\rightarrow p \pi^{-}\right) \gamma\right) K^{-} / \pi^{-}$is a level-3 cascade decay with the angular distribution, given by

$$
\begin{aligned}
\mathcal{D}_{3}(\vec{\Omega})= & \frac{1}{\Gamma} \frac{\partial^{5} \Gamma}{\partial \cos \theta \partial \cos \theta_{1} \partial \cos \theta_{2} \partial \phi \partial \phi_{1}} \\
= & \frac{1}{32 \pi^{2}}\left[1-P_{b} \alpha_{\Lambda} \cos \theta \cos \theta_{1} \cos \theta_{2}+\alpha_{b}\left(P_{b} \cos \theta-\alpha_{\Lambda} \cos \theta_{1} \cos \theta_{2}\right)\right. \\
& \left.+P_{b} \alpha_{\Lambda}\left(\gamma^{\prime} \cos \phi-\beta \sin \phi\right) \sin \theta \sin \theta_{1} \cos \theta_{2}\right]
\end{aligned}
$$

where the definitions of $\theta, \theta_{1}$ and $\phi$ are same as the previous ones, while $\phi_{1}\left(\theta_{2}\right)$ stands for the polar (azimuthal) angle in the level-2 (3) decays, shown in figures $2 \mathrm{c}$ and $2 \mathrm{~d}$. Due to the angular momentum conservation, $\mathcal{D}_{3}(\vec{\Omega})$ is independent of $\phi_{1}$.

Similar to $\mathcal{D}_{2}(\vec{\Omega}), \beta$ are also followed by $P_{b}$ in $\mathcal{D}_{3}(\vec{\Omega})$, which is a sensible result. Notably, to observe $\beta$, it is necessary to include the cascade decays of $\Lambda$. To see this, by integrating $\mathcal{D}_{3}(\vec{\Omega})$ over $\phi_{1}$ and $\cos \theta_{2}$, we find that

$$
\int_{0}^{2 \pi} \int_{-1}^{1} \mathcal{D}_{3}(\vec{\Omega}) d \cos \theta_{2} d \phi_{1}=\frac{1}{8 \pi}\left(1+\alpha_{b} P_{b} \cos \theta\right)
$$

which is identical to $\mathcal{D}_{2}(\Omega)$ by setting $\alpha=0$. Due to this reason, as opposed to $\mathcal{D}_{2}(\vec{\Omega})$, it is not sufficient to merely consider the $\phi$-dependence for observing TVEs, and there is no simple $\mathrm{T}$ violating observable as the one defined in eq. (3.3).

Note that $\alpha_{\Lambda, \Sigma^{+}}$can be determined by the experiments in the hyperon decays, given by $[29,68]$

$$
\alpha_{\Lambda}=0.731 \pm 0.014, \quad \alpha_{\Sigma^{+}}=-0.982 \pm 0.014 .
$$

However, the values of $P_{b}$ depend on the production method of the bottom baryons, which are $\mathcal{O}(10 \%)$ at $\mathrm{LHCb}[38,69]$. Theoretically, it has been suggested that $P_{b}$ can be as large as $20 \%$ [58]. Their actual values could be measured from the decays with enough data points [70, 71]. For instances, we can obtain $P_{b}$ from the angular distributions of $\Xi_{b}^{0,+} \rightarrow \Xi^{0,+} J / \psi\left(\rightarrow l^{+} l^{-}\right)$, similar to $\Lambda_{b} \rightarrow \Lambda J / \psi\left(\rightarrow l^{+} l^{-}\right)$[36-38], in which $P_{b}$ is found to be $[-0.06,0.05],[-0.04,0.05]$ and $[-0.01,0.07]$ at the central energies of 7,8 and $13 \mathrm{TeV}$ of proton-proton collisions, respectively, with $68 \%$ credibility level intervals [38]. Finally, we note that the branching ratios of $\Xi_{b}^{0,+} \rightarrow \Xi^{0,+} J / \psi$ are found to be around $10^{-4}$ in terms of the $\mathrm{SU}(3)$ flavor symmetry with the experimental input of $\mathcal{B}\left(\Lambda_{b} \rightarrow \Lambda J / \psi\right)=$ $(5.8 \pm 0.8) \times 10^{-5}[29]$. A large decay branching ratio can enhance the experiments to extract $P_{b}$ with high precision.

\section{Conclusions}

Aiming on probing TV, we have analyzed the most simple cases in the charmless twobody decays of $\mathbf{B}_{b} \rightarrow \mathbf{B}_{n} M$ with $M=(P, V, \gamma)$, in which the TVEs are described by the T-odd spin correlations. We have found that the $\mathrm{T}$ violating parameters of 
$\beta_{w}\left(\Xi_{b}^{0,-} \rightarrow \Sigma^{+, 0} K^{-}, \Xi_{b}^{-, 0} \rightarrow\left(\Lambda^{0}, p\right) K^{-}\right)$and $\beta_{w}\left(\Xi_{b}^{0,-} \rightarrow \Sigma^{+, 0} \pi^{-}, \Xi_{b}^{-, 0} \rightarrow\left(\Lambda^{0}, p\right) \pi^{-}\right)$are around $-40 \%$ and $25 \%$ with small uncertainties in the standard model, which can be measured by the experiments at $\mathrm{LHCb}$, respectively. We emphasize that in contrast to other $\mathrm{CP}$ asymmetries, the signs of $\beta_{w}$ are insensitive to the strong phases which can not be reliably calculated. Hence, if the experiments obtain opposite signs of $\beta_{w}$, it would be a smoking gun for new physics, such as the right-handed current beyond the standard model.

We have studied the angular distributions of the $\Xi_{b}$ cascade decays and identified the TVEs, in which polarized $\Xi_{b}$ are needed in order to observe TV. We have also shown that $P_{b}$ can be obtained from the angular distributions of $\Xi_{b} \rightarrow \Xi J / \psi\left(\rightarrow l^{+} l^{-}\right)$in the experiments at $\mathrm{LHCb}$ since their decay branching ratios are predicted to be around $10^{-4}$ by virtual of the $\mathrm{SU}(3)$ flavor symmetry.

Open Access. This article is distributed under the terms of the Creative Commons Attribution License (CC-BY 4.0), which permits any use, distribution and reproduction in any medium, provided the original author(s) and source are credited.

\section{References}

[1] J.S. Schwinger, The Theory of quantized fields. 1, Phys. Rev. 82 (1951) 914 [INSPIRE].

[2] J.H. Christenson, J.W. Cronin, V.L. Fitch and R. Turlay, Evidence for the $2 \pi$ Decay of the $K_{2}^{0}$ Meson, Phys. Rev. Lett. 13 (1964) 138 [INSPIRE].

[3] BeLLE collaboration, Observation of large CP-violation in the neutral B meson system, Phys. Rev. Lett. 87 (2001) 091802 [hep-ex/0107061] [InSPIRE].

[4] BABAR collaboration, Measurement of CP-violating asymmetries in $B^{0}$ decays to $C P$ eigenstates, Phys. Rev. Lett. 86 (2001) 2515 [hep-ex/0102030] [INSPIRE].

[5] BABAR collaboration, Observation of Time Reversal Violation in the $B^{0}$ Meson System, Phys. Rev. Lett. 109 (2012) 211801 [arXiv:1207.5832] [INSPIRE].

[6] G. Valencia, Angular Correlations in the Decay $B \rightarrow V V$ and CP Violation, Phys. Rev. D 39 (1989) 3339 [INSPIRE].

[7] G. Bélanger and C.Q. Geng, T violating muon polarization in K(mu-3) decays, Phys. Rev. D 44 (1991) 2789 [INSPIRE].

[8] P. Agrawal, J.N. Ng, G. Bélanger and C.Q. Geng, CP violation in $\mathrm{K}^{+} \rightarrow \pi^{+}$lepton anti-lepton decays, Phys. Rev. Lett. 67 (1991) 537 [INSPIRE].

[9] P. Agrawal, J.N. Ng, G. Bélanger and C.Q. Geng, A Study of T violation in $K^{+} \rightarrow \pi^{+} \mu^{+} \mu^{-}$ decays, Phys. Rev. D 45 (1992) 2383 [inSPIRE].

[10] G. Kramer, W.F. Palmer and H. Simma, CP violation and strong phases from penguins in $B^{ \pm} \rightarrow V V$ decays, Nucl. Phys. B 428 (1994) 77 [hep-ph/9402227] [INSPIRE].

[11] A. Ali, G. Kramer and C.-D. Lu, CP violating asymmetries in charmless nonleptonic decays $B \rightarrow P P, P V, V V$ in the factorization approach, Phys. Rev. D 59 (1999) 014005 [hep-ph/9805403] [INSPIRE].

[12] C.-W. Chiang and L. Wolfenstein, Observables in the decays of $B$ to two vector mesons, Phys. Rev. D 61 (2000) 074031 [hep-ph/9911338] [INSPIRE]. 
[13] C.Q. Geng and J.N. Ng, Testing discrete symmetries with the decay $\eta \rightarrow \pi^{+} \pi^{-} \gamma$, Phys. Scripta T 99 (2002) 109.

[14] C.-H. Chen and C.Q. Geng, Analysis of $B \rightarrow K^{*} \ell^{+} \ell^{-}$decays at large recoil region, Nucl. Phys. B 636 (2002) 338 [hep-ph/0203003] [INSPIRE].

[15] A.L. Kagan, Polarization in $B \rightarrow V V$ decays, Phys. Lett. B 601 (2004) 151 [hep-ph/0405134] [INSPIRE].

[16] A. Datta and D. London, Triple-product correlations in $B \rightarrow V_{1} V_{2}$ decays and new physics, Int. J. Mod. Phys. A 19 (2004) 2505 [hep-ph/0303159] [InSPIRE].

[17] D. London, N. Sinha and R. Sinha, Bounds on new physics from $B \rightarrow V_{1} V_{2}$ decays, Phys. Rev. D 69 (2004) 114013 [hep-ph/0402214] [INSPIRE].

[18] A. Datta, Y. Gao, A.V. Gritsan, D. London, M. Nagashima and A. Szynkman, Study of Polarization in $B \rightarrow V T$ Decays, Phys. Rev. D 77 (2008) 114025 [arXiv:0711.2107] [INSPIRE].

[19] M. Gronau and J.L. Rosner, Triple product asymmetries in $K, D_{(s)}$ and $B_{(s)}$ decays, Phys. Rev. D 84 (2011) 096013 [arXiv:1107.1232] [INSPIRE].

[20] C.Q. Geng and Y.K. Hsiao, T violation in $\bar{B}^{0} \rightarrow \Lambda \bar{p} \pi^{+}$, Phys. Rev. D 72 (2005) 037901 [INSPIRE].

[21] C.Q. Geng and Y.K. Hsiao, Direct CP and T Violation in Baryonic B Decays, Int. J. Mod. Phys. A 23 (2008) 3290 [arXiv:0801.0022] [INSPIRE].

[22] C.-H. Chen, C.-Q. Geng and L. Li, New CP phase of $B_{s}-\bar{B}_{s}$ mixing on $T$ violation in $B_{d(s)} \rightarrow K^{*}(\phi) \ell^{+} \ell^{-}$, Phys. Lett. B $\mathbf{6 7 0}(2009) 374$ [arXiv:0808.0127] [INSPIRE].

[23] R.-H. Li, C.-D. Lu and W. Wang, Branching ratios, forward-backward asymmetries and angular distributions of $B \rightarrow K_{2}^{*} l^{+} l^{-}$in the standard model and new physics scenarios, Phys. Rev. D 83 (2011) 034034 [arXiv: 1012.2129] [INSPIRE].

[24] C.Q. Geng and C.-W. Liu, Searching for possible evidences of new physics in $B \rightarrow V_{1} V_{2}$, Phys. Lett. B $\mathbf{8 2 5}$ (2022) 136883 [arXiv:2106.10628] [INSPIRE].

[25] LHCb collaboration, Measurement of the polarization amplitudes and triple product asymmetries in the $B_{s}^{0} \rightarrow \phi \phi$ decay, Phys. Lett. B 713 (2012) 369 [arXiv:1204.2813] [INSPIRE].

[26] LHCb collaboration, Measurement of polarization amplitudes and CP asymmetries in $B^{0} \rightarrow \phi K^{*}(892)^{0}$, JHEP 05 (2014) 069 [arXiv: 1403.2888] [INSPIRE].

[27] LHCb collaboration, Study of the $B^{0} \rightarrow \rho(770)^{0} K^{*}(892)^{0}$ decay with an amplitude analysis of $B^{0} \rightarrow\left(\pi^{+} \pi^{-}\right)\left(K^{+} \pi^{-}\right)$decays, JHEP 05 (2019) 026 [arXiv: 1812.07008] [INSPIRE].

[28] LHCb collaboration, Measurement of CP-violation in the $B_{s}^{0} \rightarrow \phi \phi$ decay and search for the $B^{0} \rightarrow \phi \phi$ decay, JHEP 12 (2019) 155 [arXiv: 1907.10003] [INSPIRE].

[29] Particle Data Group collaboratoin, Review of Particle Physics, PTEP 2020 (2020) 083C01 [inSPIRE].

[30] LHCb collaboration, Precision measurement of the mass and lifetime of the $\Xi_{b}^{0}$ baryon, Phys. Rev. Lett. 113 (2014) 032001 [arXiv:1405.7223] [INSPIRE].

[31] LHCb collaboration, Precision Measurement of the Mass and Lifetime of the $\Xi_{b}^{-}$Baryon, Phys. Rev. Lett. 113 (2014) 242002 [arXiv:1409.8568] [InSPIRE]. 
[32] CMS collaboration, Measurement of b hadron lifetimes in pp collisions at $\sqrt{s}=8 \mathrm{TeV}$, Eur. Phys. J. C 78 (2018) 457 [Erratum ibid. 78 (2018) 561] [arXiv:1710.08949] [InSPIRE].

[33] LHCb collaboration, Observation of the decay $\Xi_{b}^{-} \rightarrow p K^{-} K^{-}$, Phys. Rev. Lett. 118 (2017) 071801 [arXiv: 1612.02244] [INSPIRE].

[34] LHCb collaboration, Search for $C P$ violation in $\Lambda_{b}^{0} \rightarrow p K^{-}$and $\Lambda_{b}^{0} \rightarrow p \pi^{-}$decays, Phys. Lett. B 787 (2018) 124 [arXiv: 1807.06544] [INSPIRE].

[35] LHCb collaboration, Measurements of CP asymmetries in charmless four-body $\Lambda_{b}^{0}$ and $\Xi_{b}^{0}$ decays, Eur. Phys. J. C 79 (2019) 745 [arXiv: 1903.06792] [INSPIRE].

[36] ATLAS collaboration, Measurement of the parity-violating asymmetry parameter $\alpha_{b}$ and the helicity amplitudes for the decay $\Lambda_{b}^{0} \rightarrow J / \psi+\Lambda^{0}$ with the ATLAS detector, Phys. Rev. D 89 (2014) 092009 [arXiv:1404.1071] [INSPIRE].

[37] CMS collaboration, Measurement of the $\Lambda_{b}$ polarization and angular parameters in $\Lambda_{b} \rightarrow J / \psi \Lambda$ decays from pp collisions at $\sqrt{s}=7$ and 8 TeV, Phys. Rev. D 97 (2018) 072010 [arXiv: 1802.04867] [INSPIRE].

[38] LHCb collaboration, Measurement of the $\Lambda_{b}^{0} \rightarrow J / \psi \Lambda$ angular distribution and the $\Lambda_{b}^{0}$ polarisation in pp collisions, JHEP 06 (2020) 110 [arXiv:2004.10563] [INSPIRE].

[39] C.-D. Lu, Y.-M. Wang, H. Zou, A. Ali and G. Kramer, Anatomy of the pQCD Approach to the Baryonic Decays $\Lambda_{b} \rightarrow p \pi, p K$, Phys. Rev. D 80 (2009) 034011 [arXiv:0906.1479] [INSPIRE].

[40] A. Khodjamirian, C. Klein, T. Mannel and Y.M. Wang, Form Factors and Strong Couplings of Heavy Baryons from QCD Light-Cone Sum Rules, JHEP 09 (2011) 106 [arXiv: 1108.2971] [INSPIRE].

[41] T. Gutsche, M.A. Ivanov, J.G. Körner, V.E. Lyubovitskij and P. Santorelli, Polarization effects in the cascade decay $\Lambda_{b} \rightarrow \Lambda\left(\rightarrow p \pi^{-}\right)+J / \psi\left(\rightarrow \ell^{+} \ell^{-}\right)$in the covariant confined quark model, Phys. Rev. D 88 (2013) 114018 [arXiv:1309.7879] [INSPIRE].

[42] Y.K. Hsiao and C.Q. Geng, Direct CP-violation in $\Lambda_{b}$ decays, Phys. Rev. D 91 (2015) 116007 [arXiv: 1412.1899] [INSPIRE].

[43] Y.K. Hsiao, Y. Yao and C.Q. Geng, Charmless two-body anti-triplet b-baryon decays, Phys. Rev. D 95 (2017) 093001 [arXiv: 1702.05263] [INSPIRE].

[44] T. Gutsche, M.A. Ivanov, J.G. Körner and V.E. Lyubovitskij, Nonleptonic two-body decays of single heavy baryons $\Lambda_{Q}, \Xi_{Q}$, and $\Omega_{Q}(Q=b, c)$ induced by $W$ emission in the covariant confined quark model, Phys. Rev. D 98 (2018) 074011 [arXiv:1806.11549] [INSPIRE].

[45] Z.-X. Zhao, Weak decays of heavy baryons in the light-front approach, Chin. Phys. C 42 (2018) 093101 [arXiv: 1803.02292] [INSPIRE].

[46] P. Böer, A. Kokulu, J.-N. Toelstede and D. van Dyk, Angular Analysis of $\Lambda_{b} \rightarrow \Lambda_{c}(\rightarrow \Lambda \pi) \ell \bar{\nu}$, JHEP 12 (2019) 082 [arXiv:1907.12554] [INSPIRE].

[47] J. Zhu, Z.-T. Wei and H.-W. Ke, Semileptonic and nonleptonic weak decays of $\Lambda_{b}^{0}$, Phys. Rev. D 99 (2019) 054020 [arXiv: 1803.01297] [INSPIRE].

[48] C.-Q. Geng, C.-W. Liu and T.-H. Tsai, Nonleptonic two-body weak decays of $\Lambda_{b}$ in modified MIT bag model, Phys. Rev. D 102 (2020) 034033 [arXiv: 2007.09897] [INSPIRE].

[49] A. Dery, M. Ghosh, Y. Grossman and S. Schacht, $\mathrm{SU}(3)_{F}$ analysis for beauty baryon decays, JHEP 03 (2020) 165 [arXiv:2001.05397] [INSPIRE]. 
[50] J. Franklin, Quark model relations for b-baryon decay, J. Phys. G $4 \mathbf{7}$ (2020) 085001 [arXiv:2004.01037] [INSPIRE].

[51] S. Roy, R. Sinha and N.G. Deshpande, Nonleptonic beauty baryon decays and CP asymmetries based on an $\mathrm{SU}(3)$-flavor analysis, Phys. Rev. D 101 (2020) 036018 [arXiv: 1911.01121] [INSPIRE].

[52] S. Roy, R. Sinha and N.G. Deshpande, Beauty baryon nonleptonic decays into decuplet baryons and CP-asymmetries based on an $\mathrm{SU}(3)$-flavor analysis, Phys. Rev. D 102 (2020) 053007 [arXiv: 2006.16813] [INSPIRE].

[53] J.-J. Han, R.-X. Zhang, H.-Y. Jiang, Z.-J. Xiao and F.-S. Yu, Weak decays of bottom-charm baryons: $\mathcal{B}_{b c} \rightarrow \mathcal{B}_{b}$ P, Eur. Phys. J. C 81 (2021) 539 [arXiv:2102.00961] [InSPIRE].

[54] C.Q. Geng, C.W. Liu and T.H. Tsai, Non-leptonic two-body decays of $\Lambda_{b}^{0}$ in light-front quark model, Phys. Lett. B 815 (2021) 136125 [arXiv:2102.01552] [INSPIRE].

[55] W. Bensalem, A. Datta and D. London, T violating triple product correlations in charmless $\Lambda_{b}$ decays, Phys. Lett. B 538 (2002) 309 [hep-ph/0205009] [INSPIRE].

[56] W. Bensalem, A. Datta and D. London, New physics effects on triple product correlations in $\Lambda_{b}$ decays, Phys. Rev. D 66 (2002) 094004 [hep-ph/0208054] [INSPIRE].

[57] C.-H. Chen, C.Q. Geng and J.N. Ng, T violation in $\Lambda_{b} \rightarrow \Lambda \ell^{+} \ell^{-}$decays with polarized Lambda, Phys. Rev. D 65 (2002) 091502 [hep-ph/0202103] [INSPIRE].

[58] Z.J. Ajaltouni, E. Conte and O. Leitner, $\Lambda_{b}$ decays into $\Lambda$-vector, Phys. Lett. B 614 (2005) 165 [hep-ph/0412116] [INSPIRE].

[59] O. Leitner, Z.J. Ajaltouni and E. Conte, An Angular distribution analysis of $\Lambda_{b}$ decays, Nucl. Phys. A 755 (2005) 435 [hep-ph/0412131] [INSPIRE].

[60] O. Leitner and Z.J. Ajaltouni, Testing CP and Time Reversal Symmetries with $\Lambda_{b} \rightarrow \Lambda V(1-)$ Decays, Nucl. Phys. B Proc. Suppl. 174 (2007) 169 [hep-ph/0610189] [INSPIRE].

[61] M. Gronau and J.L. Rosner, Triple product asymmmetries in $\Lambda_{b}$ and $\Xi_{b}$ decays, Phys. Lett. $B 749$ (2015) 104 [arXiv:1506.01346] [INSPIRE].

[62] C.-Q. Geng and C.-W. Liu, Time-reversal asymmetries and angular distributions in $\Lambda_{b} \rightarrow \Lambda V$, JHEP 11 (2021) 104 [arXiv:2109.09524] [INSPIRE].

[63] A.J. Buras, M. Jamin, M.E. Lautenbacher and P.H. Weisz, Effective Hamiltonians for $\Delta S=1$ and $\Delta B=1$ nonleptonic decays beyond the leading logarithmic approximation, Nucl. Phys. B 370 (1992) 69 [Addendum ibid. 375 (1992) 501] [InSPIRE].

[64] J.F. Donoghue, X.-G. He and S. Pakvasa, Hyperon Decays and CP Nonconservation, Phys. Rev. D 34 (1986) 833 [INSPIRE].

[65] L. Wolfenstein, Parametrization of the Kobayashi-Maskawa Matrix, Phys. Rev. Lett. 51 (1983) 1945 [INSPIRE].

[66] A. Ali, G. Kramer and C.-D. Lu, Experimental tests of factorization in charmless nonleptonic two-body B decays, Phys. Rev. D 58 (1998) 094009 [hep-ph/9804363] [INSPIRE].

[67] J.G. Korner and M. Krämer, Exclusive nonleptonic charm baryon decays, Z. Phys. C $\mathbf{5 5}$ (1992) 659 [INSPIRE]. 
[68] BESIII collaboration, Polarization and Entanglement in Baryon-Antibaryon Pair Production in Electron-Positron Annihilation, Nature Phys. 15 (2019) 631 [arXiv:1808.08917] [INSPIRE].

[69] G. Hiller, M. Knecht, F. Legger and T. Schietinger, Photon polarization from helicity suppression in radiative decays of polarized Lambda(b) to spin-3/2 baryons, Phys. Lett. B 649 (2007) 152 [hep-ph/0702191] [INSPIRE].

[70] BESIII collaboration, Precision measurement of the $e^{+} e^{-} \rightarrow \Lambda_{c}^{+} \bar{\Lambda}_{c}^{-}$cross section near threshold, Phys. Rev. Lett. 120 (2018) 132001 [arXiv:1710.00150] [InSPIRE].

[71] BESIII collaboration, Measurements of Weak Decay Asymmetries of $\Lambda_{c}^{+} \rightarrow p K_{S}^{0}, \Lambda \pi^{+}$, $\Sigma^{+} \pi^{0}$, and $\Sigma^{0} \pi^{+}$, Phys. Rev. D 100 (2019) 072004 [arXiv: 1905.04707] [INSPIRE]. 